\title{
The mitochondrial heme exporter FLVCR1b mediates erythroid differentiation
}

\author{
Deborah Chiabrando, ${ }^{1}$ Samuele Marro, ${ }^{1,2}$ Sonia Mercurio, ${ }^{1}$ Carlotta Giorgi, ${ }^{3}$ Sara Petrillo, ${ }^{1}$ \\ Francesca Vinchi, ${ }^{1}$ Veronica Fiorito, ${ }^{1}$ Sharmila Fagoonee, ${ }^{1}$ Annalisa Camporeale, ${ }^{1}$ Emilia Turco, ${ }^{1}$ \\ Giorgio R. Merlo, ${ }^{1}$ Lorenzo Silengo, ${ }^{1}$ Fiorella Altruda, ${ }^{1}$ Paolo Pinton, ${ }^{3}$ and Emanuela Tolosano ${ }^{1}$

\begin{abstract}
${ }^{1}$ Molecular Biotechnology Centre, Department of Genetics, Biology and Biochemistry, University of Torino, Torino, Italy.
${ }^{2}$ Institute for Stem Cell Biology and Regenerative Medicine, Stanford University School of Medicine, Stanford, California, USA.

${ }^{3}$ Department of Experimental and Diagnostic Medicine, Section of General Pathology, Interdisciplinary Center for the Study of Inflammation (ICSI),
\end{abstract} \\ Laboratory for Technologies of Advanced Therapies (LTTA), University of Ferrara, Ferrara, Italy.
}

\begin{abstract}
Feline leukemia virus subgroup C receptor 1 (FLVCR1) is a cell membrane heme exporter that maintains the balance between heme levels and globin synthesis in erythroid precursors. It was previously shown that Flvcr1null mice died in utero due to a failure of erythropoiesis. Here, we identify Flvcr $1 b$, a mitochondrial Flver1 isoform that promotes heme efflux into the cytoplasm. Flvcr $1 b$ overexpression promoted heme synthesis and in vitro erythroid differentiation, whereas silencing of Flvcr $1 b$ caused mitochondrial heme accumulation and termination of erythroid differentiation. Furthermore, mice lacking the plasma membrane isoform (Flvcr1a) but expressing Flvcr $1 b$ had normal erythropoiesis, but exhibited hemorrhages, edema, and skeletal abnormalities. Thus, FLVCR1b regulates erythropoiesis by controlling mitochondrial heme efflux, whereas FLVCR1a expression is required to prevent hemorrhages and edema. The aberrant expression of Flver 1 isoforms may play a role in the pathogenesis of disorders characterized by an imbalance between heme and globin synthesis.
\end{abstract}

\section{Introduction}

Erythropoiesis is a tightly controlled process regulated by both systemic and cellular signals. Among the latter, heme plays a crucial role not only because it is an essential structural component of hemoglobin, but also because it regulates both the transcription and translation of globin chains through interaction with the transcriptional repressor Btb and Cnc homology 1 (BACH1) and the heme-regulated eIF $2 \alpha$ kinase (HRI) respectively $(1,2)$. Recently, heme export through the cell-surface transporter feline leukemia virus subgroup C receptor 1 (FLVCR1) was proposed as an additional control step in this process to prevent intracellular heme overload (3, 4). Consistently, Flvcr1-knockout mice die in utero due to a block of erythroid differentiation at the proerythroblast stage, suggesting that heme export at earlier stages of erythroid differentiation may contribute to maintain the correct stoichiometry between heme and globin levels $(3,4)$.

Heme is endogenously synthesized through 8 conserved enzymatic reactions, starting in the mitochondrial matrix with the condensation of succinyl-CoA and glycine to form $\delta$-aminolevulinic acid (ALA), a reaction catalyzed by ALA synthase 2 (ALAS2) in erythroid cells and ALAS1 in other cell types (5). Following its synthesis, ALA is exported to the cytosol, where it is converted through a series of enzymatic reactions to coproporphyrinogen III (CPgenIII). Then, this molecule is imported back to the mitochondrion and converted to protoporphyrin IX (PPIX). Finally, ferrous iron is incorporated into PPIX in the mitochondrial matrix, a reaction catalyzed by ferrochelatase $(\mathrm{FECH})(6)$. While all the enzymatic steps leading to the production of heme are well characterized, it is still not completely understood how the compartmentalization of these reactions between the mitochondrion and cytosol

Authorship note: Samuele Marro and Sonia Mercurio contributed equally to this work.

Conflict of interest: The authors have declared that no conflict of interest exists. Citation for this article: J Clin Invest. 2012;122(12):4569-4579. doi:10.1172/JCI62422. occurs, how heme is exported out of the mitochondrion, and how heme efflux from this organelle is regulated $(7,8)$. Recently, the ATP-binding cassette transporter ABCB6 has been proposed as a porphyrin importer into the mitochondrion (9), but no mitochondrial heme exporter has been identified to date.

In this work, we identify Flvcrlb as a mitochondrial isoform of Flver1 that localizes to the mitochondrion and that, by controlling heme efflux from this organelle, plays an essential role in erythroid differentiation. The analysis of a mouse model lacking the plasma membrane isoform (herein called Flvcr1a) but still expressing Flver $1 b$ indicates that FLVCR1a is dispensable for definitive erythropoiesis, but is required to prevent hemorrhages and edema.

\section{Results}

FLVCR1b is a mitochondrial protein. The analysis of predicted transcripts for the human Flverl gene led to the identification of the expressed sequence tag (EST) CN275423, which lacks exon 1 and includes 95 bps of the first intron. This EST suggested the existence of a shorter Flvcr1 mRNA that we experimentally verified and named Flvcr1b (Supplemental Figure 1; supplemental material available online with this article; doi:10.1172/JCI62422DS1). Flvcr $1 b$ mRNA includes a coding sequence for a protein with 6 putative hydrophobic transmembrane domains of approximately $28 \mathrm{kDa}$ (Figure 1, A and B). FLVCR1b amino acid sequence corresponds to amino acids 277-555 of FLVCR1a protein.

Flvcr $1 b$ is ubiquitously expressed in mouse tissues: the highest transcript levels were found in the brain, followed by tissues with high heme synthesis rates, such as the heart, skeletal muscle, bone marrow, and spleen; lower mRNA levels were found in the kidney, duodenum, and liver (Figure 1C). Expression of Flvcr1b was also detected in human cell lines with erythroid features (K562) or not (HeLa, Caco2, Hek293) (Figure 1D).

When overexpressed in vitro, FLVCR1b showed a striking different sub-cellular localization compared with the previously identified membrane protein, which was named FLVCR1a (refs. 10, 11, 
and Supplemental Figure 2). A putative aminoterminal mitochondrial targeting sequence (MTS) with a cleavage site at position 38 of FLVCR1b was identified using MitoProtII software (12). Accordingly, Western blot analysis demonstrated that the myc-tagged FLVCR1b protein (FLVCR1b-myc) was specifically expressed in the mitochondrial-enriched fraction (Figure 1E). Furthermore, confocal microscopy analysis showed the colocalization between FLVCR1b-myc and the $\alpha$ subunit of the mitochondrial trifunctional protein HADHA (Figure 1F). The same result was obtained following the overexpression of the GFP-tagged FLVCR1b protein (D. Chiabrando, unpublished observations).

To support our in silico prediction concerning the identification of an MTS in FLVCR1b protein, the ability of this sequence to direct GFP expression into the mitochondrion was analyzed. To this end, GFP was fused to the C terminus of the MTS of FLVCR1b (MTS-GFP), and immunofluorescence studies were performed. Following its overexpression in HEK293 cells, MTS-GFP colocalized with MitoTracker, indicating that FLVCR1b MTS is sufficient to direct GFP expression into the mitochondrion (Supplemental Figure 3).

Based on these preliminary findings, further biochemical approaches were used to dissect the intracellular localization of the endogenous FLVCR1b protein. We took advantage of a previously developed method to further purify the mitochondria-containing fraction (crude mitochondria) through a Percoll-based centrifugation protocol into a "pure" mitochondrial fraction free of ER and nuclear proteins (13). Greater enrichment for mitochondrial proteins in the pure mitochondrial fraction was confirmed by detecting high levels of voltage-dependent anion channel (VDAC). When probed with an antibody against the $\mathrm{C}$ terminus of the human FLVCR1 protein, a specific band of the expected size for FLVCR1b was detected only in the pure mitochondrial fraction (Figure 1G).

Additionally, a detailed subcellular fractionation of mitochondria, ER, mitochondria-associated membranes (MAMs), and cytosol was performed. Consistently, FLVCR1b protein was detected only in the pure mitochondrial fraction (Figure $1 \mathrm{H}$ ), confirming the exclusive mitochondrial localization of the FVLCR1b protein.

Taken together, these data demonstrate that FLVCR1b is expressed in the mitochondrial compartment and suggest a different role for FLVCR1b in intracellular heme trafficking compared with FLVCR1a.

FLVCR1b overexpression results in intracellular heme accumulation. Due to structure similarities between FLVCR1a and FLVCR1b, we hypothesized that FLVCR1b could be a heme exporter on the mitochondrion. As an initial screen to determine whether FLVCR1b could be involved in mitochondrial heme trafficking, intracellular heme concentration was measured in HeLa cells 72 hours following the overexpression of FLVCR1b-myc, FLVCR1a-myc or a control vector (Figure 1B). As already reported (3), intracellular heme levels slightly but significantly decreased following the overexpression of the membrane heme exporter FLVCR1a compared with control cells (Figure 2A). Conversely, the overexpression of the mitochondrial isoform FLVCR1b in HeLa cells resulted in a 2 -fold increase of intracellular heme content (Figure 2A); heme increase was also indirectly demonstrated by the strong induction of the heme degrading enzyme heme oxygenase 1 (Ho-1) mRNA (Figure 2B). Succinylacetone, a competitive inhibitor of the second enzyme of the heme biosynthetic pathway (ALA dehydratase [ALAD]), completely prevented intracellular accumulation of heme observed when FLVCR1b was overexpressed (Figure 2A), indicating that heme biosynthesis was responsible for the eleva- tion of intracellular heme content. Moreover, HeLa cells overexpressing FLVCR1b showed an alteration of heme biosynthesis enzymes and transporters. Real-time PCR analysis showed a statistically significant increase of Alas1 (Figure 2C), Hmbs, Uros, Cpox, and the ATP-binding cassette transporter Abcb6 mRNA (Supplemental Figure 4A). These data are consistent with a putative role of FLVCR1b in the heme biosynthetic pathway.

Intracellular free-heme is highly toxic to cells, promoting lipid peroxidation and the production of ROS (14). HeLa cells overexpressing FLVCR1b showed increased expression of superoxide dismutase 1 (Sod-1), an oxidative stress marker (Supplemental Figure 4B). In addition to the upregulation of $\mathrm{Ho}-1$, HeLa cells overexpressing FLVCR1b had also increased Flvcrla and ATP-binding cassette subfamily G member 2 ( $A b c g 2$ ) mRNA levels (Supplemental Figure 4C) likely as a compensatory mechanism to counteract the toxic effects of free heme. Therefore, increased heme content in FLVCR1b-overexpressing cells generates oxidative stress and activates detoxifying mechanisms, such as the upregulation of heme degradation and promotion of heme export out of the cell through FLVCR1a and ABCG2.

FLVCR1b silencing results in heme accumulation in mitochondria. To investigate the consequences of the loss of FLVCR1b on heme biosynthesis, silencing experiments were performed. Cells lacking only FLVCR1a were compared with those depleted for both FLVCR1a and FLVCR1b (Supplemental Figure 5).

Loss of FLVCR1a did not affect heme content in HeLa cells because of compensatory mechanisms such as the induction of heme degradation by HO1 (Figure 2, D and E). Following the stimulation of heme synthesis by the heme precursor ALA, heme levels strongly increased in FLVCR1a-deficient HeLa cells compared with controls, despite the strong induction of $\mathrm{Ho}-1$ (Figure 2, $\mathrm{D}$ and $\mathrm{E}$ ), supporting the important role of FLVCR1a as a heme exporter at the cell membrane (3). Surprisingly, a slight but significant increase of intracellular heme content was observed in FLVCR1aand FLVCR1b-depleted HeLa cells compared with FLVCR1a deficient or control cells (Figure 2D). Interestingly, Ho-1 was not induced in these conditions (Figure 2E), implying that heme overload did not occur into the cytosol but in some intracellular compartment. To specifically address this issue, heme content was measured in mitochondria and in the cytosol. Only when the expression of both the isoforms was lost, mitochondrial heme overload was observed, while cytosolic heme content was unaltered (Figure 2F), thus indicating that FLVCR1b expression could regulate heme export out of the mitochondrion. Finally, heme content of HeLa cells lacking both FLVCR1a and FLVCR1b was comparable under basal conditions and following ALA treatment (Figure 2D), indicating that heme biosynthesis was completely impaired in these cells.

To further confirm the ability of FLVCR1b to regulate heme efflux from mitochondria, a specific siRNA against the 5' UTR of Flvcr $1 b$ was designed. Quantitative RT-PCR (qRT-PCR) analysis confirmed a reduction of Flvcr $1 b$ mRNA 72 hours after siRNA transfection, with no effect on Flvcr1a (Supplemental Figure 6). According to previous results, the specific silencing of FLVCR1b led to heme accumulation in mitochondria, while cytosolic heme levels were not affected compared with controls (Figure $2 \mathrm{G}$ ).

Finally, to rule out the possibility that the increase of mitochondrial heme content observed in FLVCR1b-deficient HeLa cells was secondary to changes in electron transport chain (ETC) enzymes, the amount of cytochrome $c$ oxidase (a component of complex IV) 
A
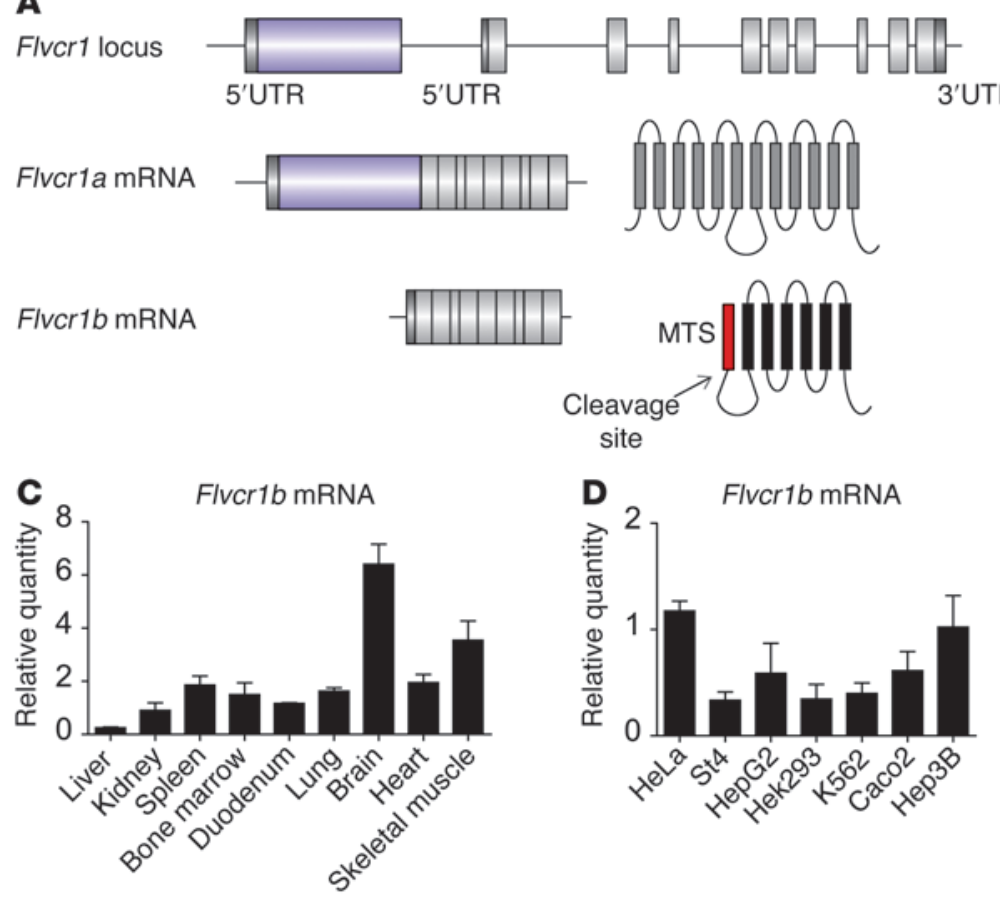

$\mathbf{F}$

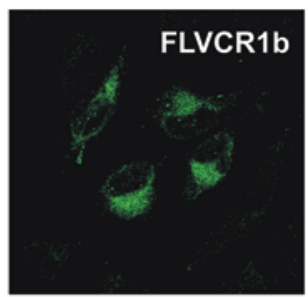

G

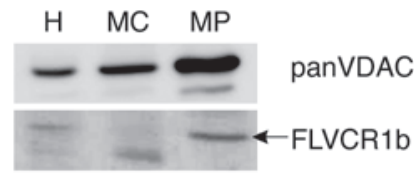

D Flvcr1b mRNA

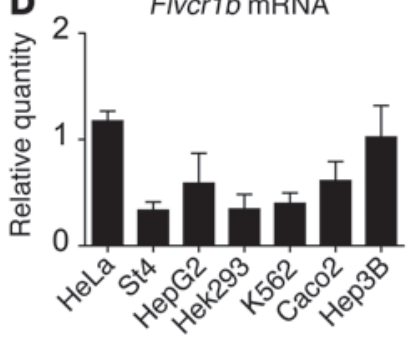

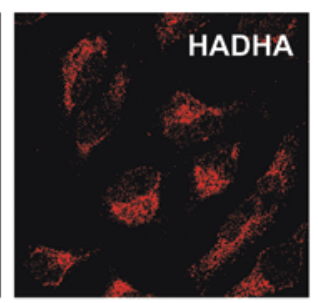

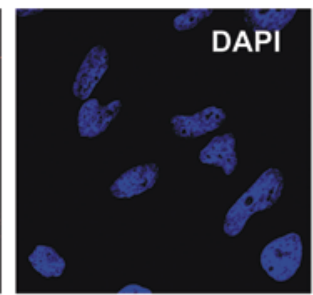

H H MC MP ER MAMCYT
B

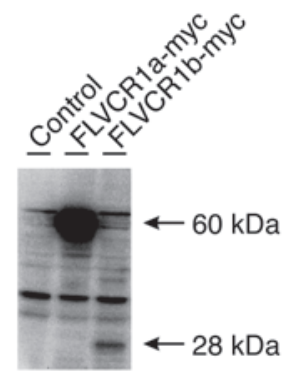

E

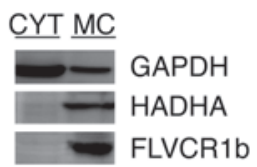

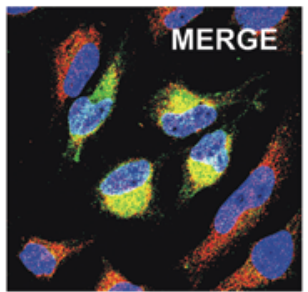

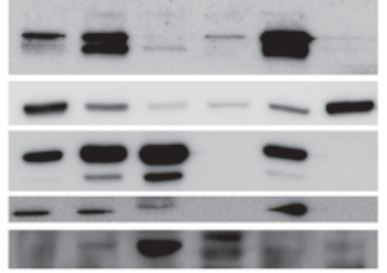

IP3R3

$\beta$-Tubulin

panVDAC

SigmaR1

FLVCR1b

\section{Figure 1}

Identification of a mitochondrial isoform of Flvcr1. (A) Schematic representation of Flvcr1 genetic locus, Flvcr1a and Flvcr1b transcripts, and the predicted protein structures. The MTS in FLVCR1b is indicated in red; cleavage at position 38 results in a protein with 6 hydrophobic transmembrane domains. See also Supplemental Figure 1. (B) Western blot analysis of total protein extracts of HeLa cells overexpressing FLVCR1a-myc, FLVCR1b-myc, or the control vector. Antibody against myc was used. (C) qRT-PCR analysis of Flvcr1b mRNA levels in mouse tissues and (D) human cell lines. Values represent mean \pm SEM. $n=6$. (E) Western blot analysis of fractionated protein extracts of HeLa cells overexpressing FLVCR1b-myc showing that FLVCR1b is localized in the mitochondrial enriched fraction. Antibodies against GAPDH (cytosolic marker), HADHA (mitochondrial marker), and myc (to detect FLVCR1b) were used. (F) Immunofluorescence analysis of HeLa cells overexpressing FLVCR1b-myc showing the colocalization between FLVCR1b (detected with an antibody against myc) and HADHA. See also Supplemental Figures 2 and 3. Original magnification, $\times 63$. (G) Endogenous FLVCR1b mitochondrial localization revealed by immunoblot analysis. Homogenate, crude, and pure mitochondrial fraction, respectively, before and after Percoll gradient separation prepared from HeLa cells. $20 \mu \mathrm{g}$ of proteins were loaded on $15 \%$ SDS-polyacrylamide gels. (H) Detection of endogenous FLVCR1b by immunoblotting in HEK293 fractionation. IP3R3 and SigmaR1 were used as markers of MAM, tubulin as a marker of cytosol, and VDAC as a marker of mitochondria. $50 \mu \mathrm{g}$ of proteins were loaded on $15 \%$ SDSpolyacrylamide gels. $\mathrm{H}$, homogenate; MC, crude mitochondria; MP,pure mitochondria; CYT, cytosol. 
A

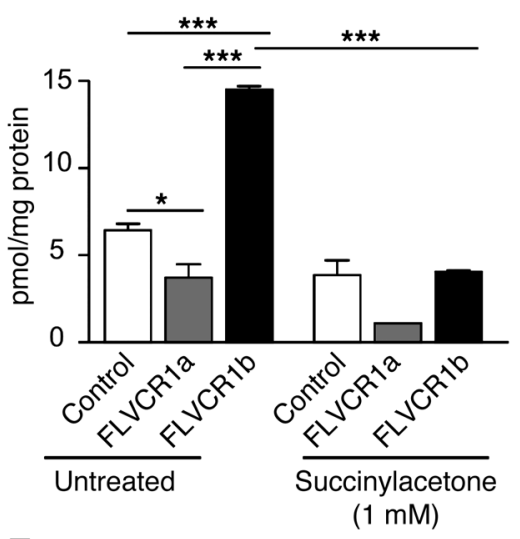

E
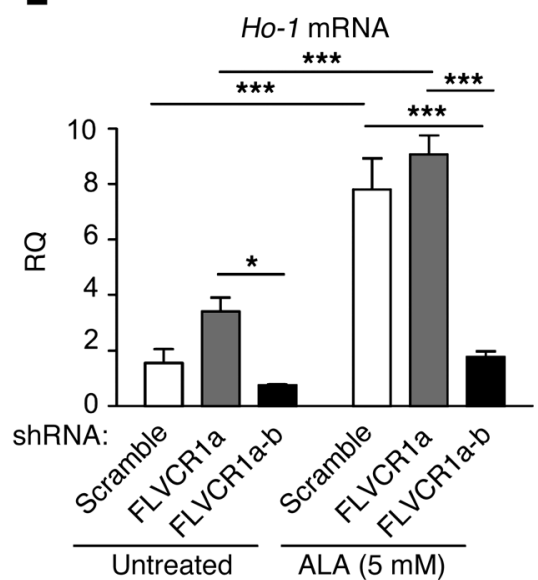

B

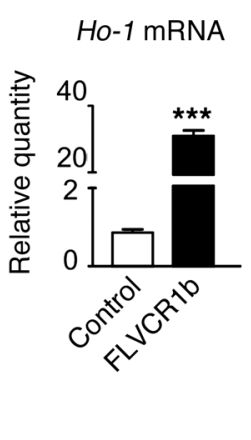

C

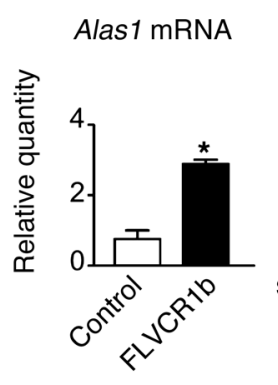

F
D

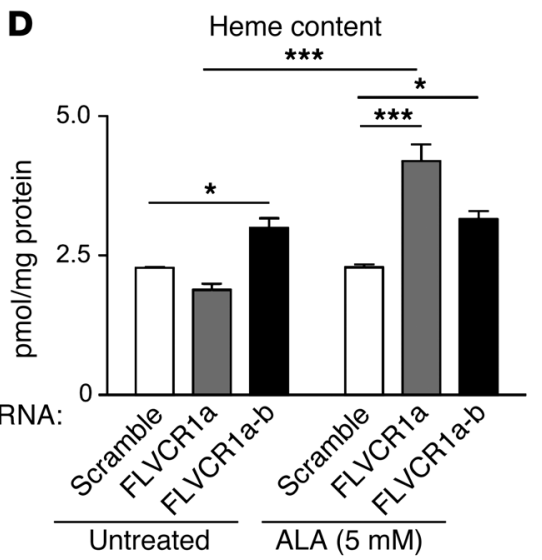

Heme content

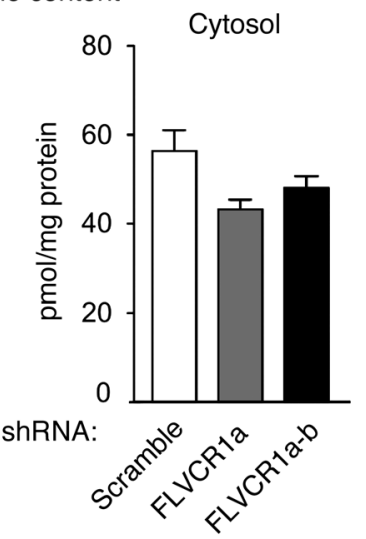

G

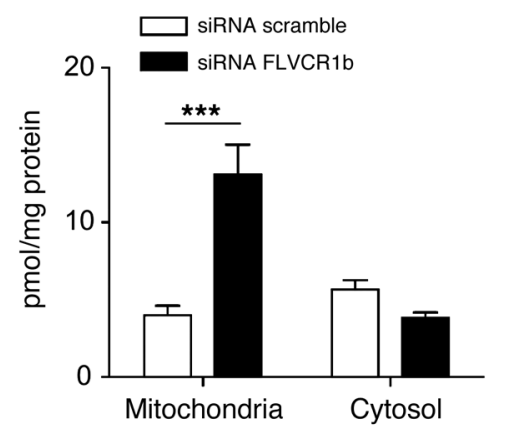

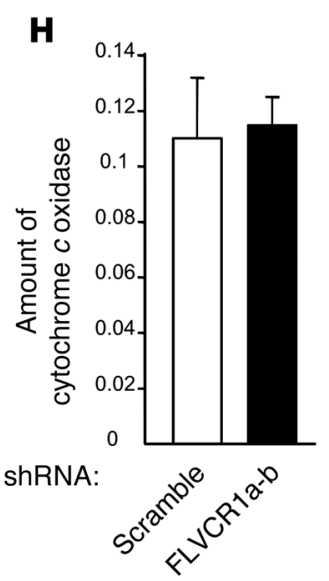

I
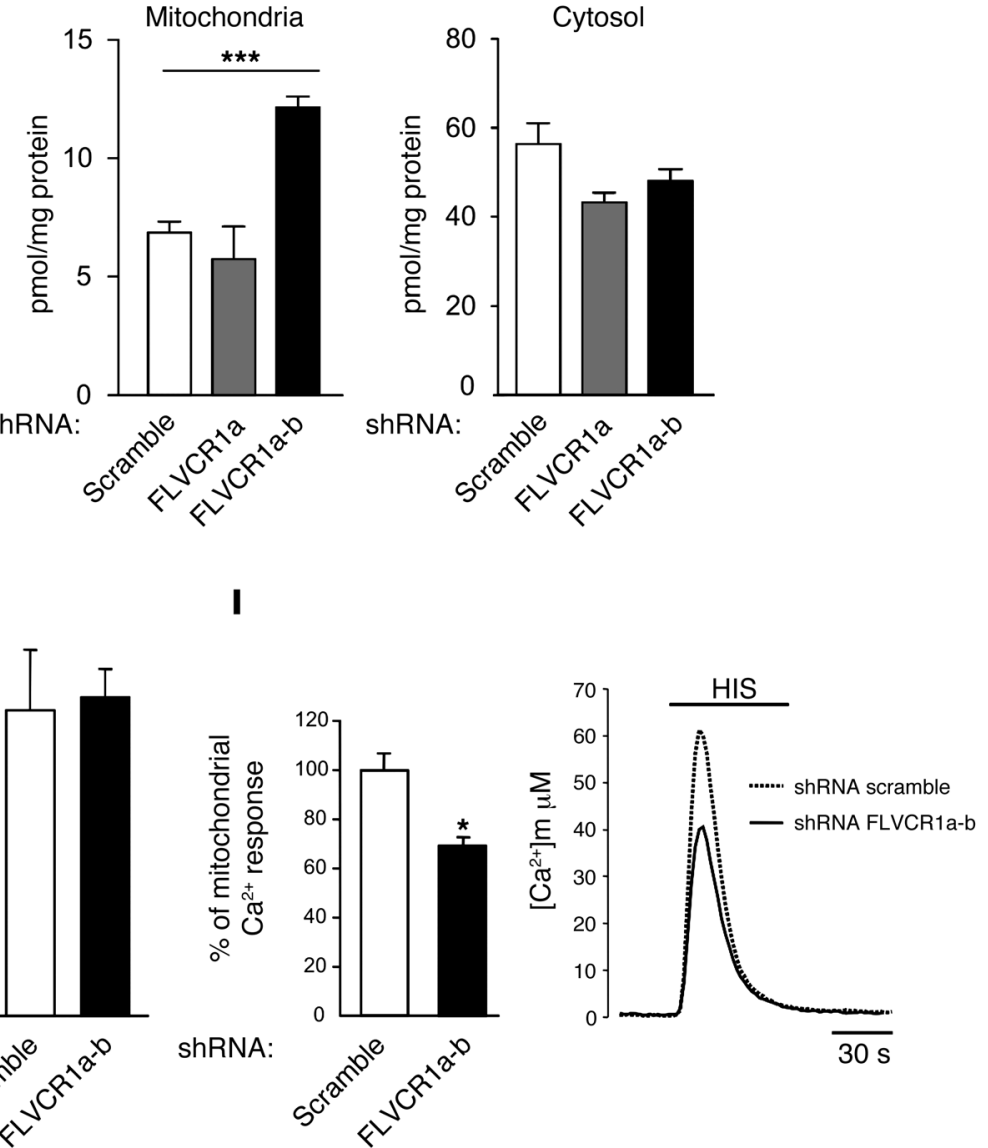

Figure 2

FLVCR1b overexpression increases intracellular heme content, while its silencing results in heme accumulation in mitochondria. (A) Heme levels in HeLa cells overexpressing FLVCR1a-myc, FLVCR1b-myc, or a control vector. Succinylacetone was used as a competitive inhibitor of heme biosynthesis. $n=6$. Two-way ANOVA. (B and C) qRT-PCR analysis of Ho-1 mRNA and Alas1 mRNA in FLVCR1b-overexpressing HeLa cells compared with control. $n=6 . t$ test. ( $\mathbf{D}$ and $\mathbf{E}$ ) Heme content and qRT-PCR analysis of Ho-1 transcript levels in HeLa cells in which the expression of both FLVCR1a and FLVCR1b, or FLVCR1a alone, was downregulated using specific shRNA. Heme biosynthesis was stimulated with ALA. $n=6$. Two-way ANOVA. (F) Heme content in mitochondria and cytosol from HeLa cells, in which the expression of both FLVCR1a and FLVCR1b, or FLVCR1a alone, was downregulated using specific shRNA compared with control cells. $n=6$. One-way ANOVA. (G) Heme content in mitochondria and cytosol isolated from HeLa cells in which FLVCR1b expression was downregulated using a specific siRNA against the 5' UTR of Flvcr1b with respect to controls. $n=6$. Two-way ANOVA. (H) Quantity of cytochrome $c$ oxidase in control versus silenced FLVCR1a-b HeLa cells. $n=4$. (I) Mitochondrial $\mathrm{Ca}^{2+}$ responses to agonist stimulation in control and silenced FVLCR1a-b HeLa cells. Data were normalized to mean of the control group. Traces are representative of 12 experiments from 3 preparations. $t$ test. Values represent mean \pm SEM. ${ }^{*} P<0.05 ;{ }^{* \star *} P<0.001$. 
A

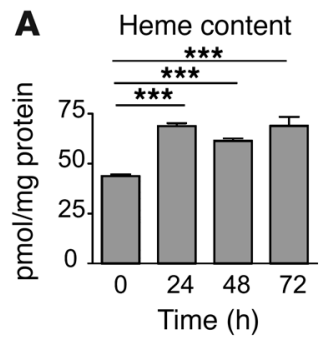

B

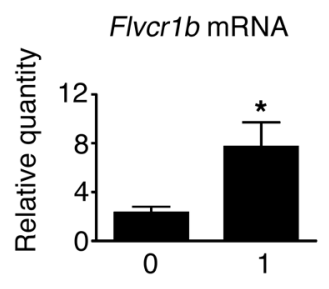

Time after phlebotomy (d)

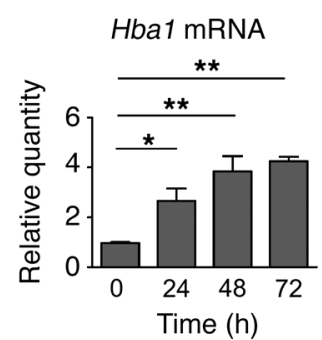

Time (h)

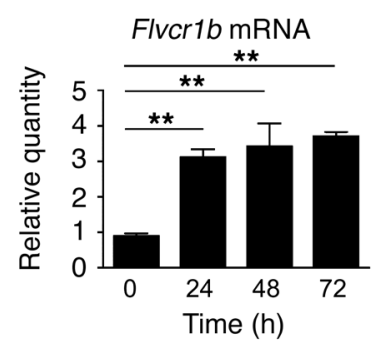

C

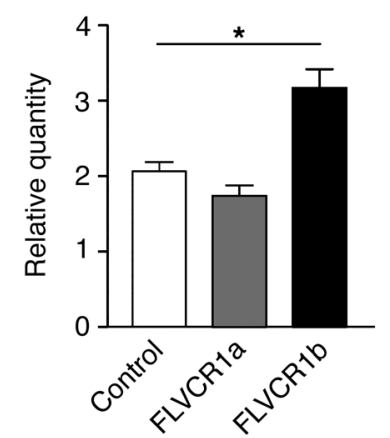

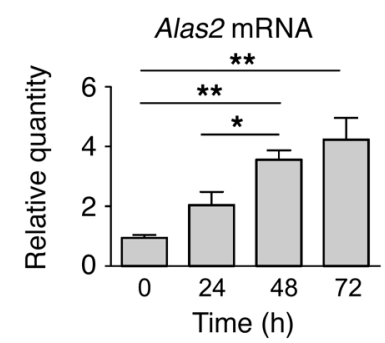

D

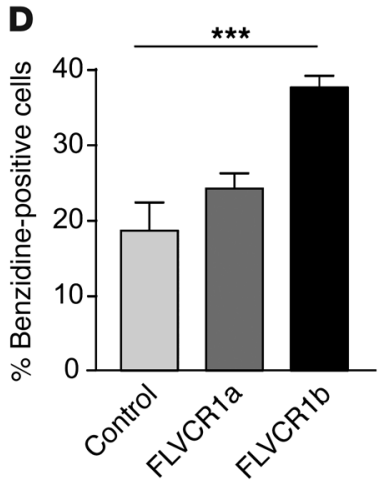

E

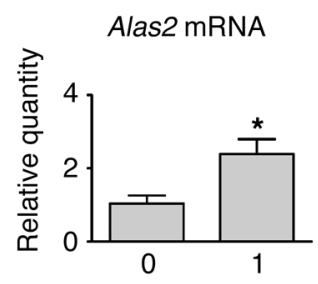

Time after phlebotomy (d)

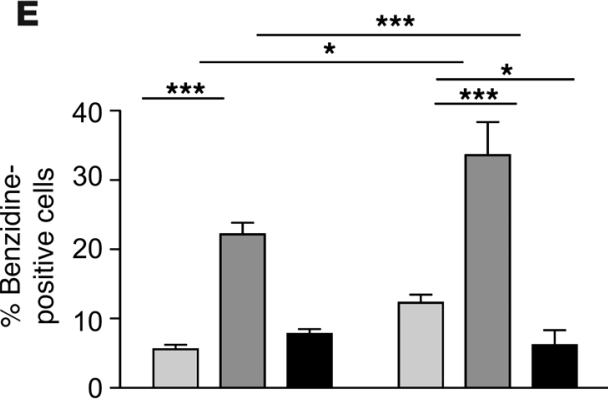

ShRNA:

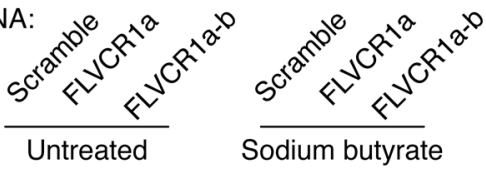

(0.5 mM)

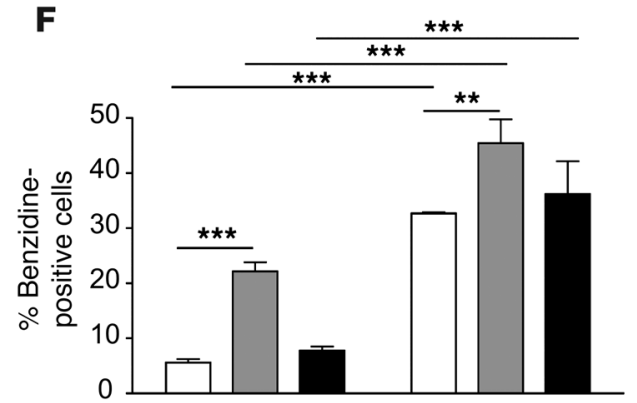

shRNA:
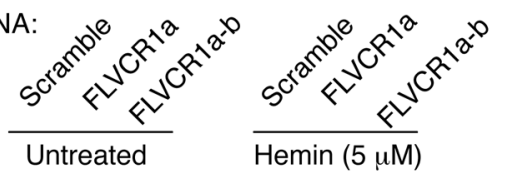

Figure 3

FLVCR1b controls erythroid differentiation in vitro. (A) Heme content and qRT-PCR analysis of Hba1, Flvcr1b, and Alas2 mRNAs in K562 cells treated with $5 \mathrm{mM}$ ALA to induce in vitro erythroid differentiation. $n=6$. One-way ANOVA. (B) qRT-PCR analysis of spleen F/vcr1b and Alas2 mRNA levels following the stimulation of erythropoiesis by phlebotomy. $n=6 . t$ test. (C) qRT-PCR analysis of Hba1 mRNA and (D) benzidine staining of K562 cells overexpressing FLVCR1a-myc, FLVCR1b-myc, or a control vector. $n=6$. One-way ANOVA. (E) Benzidine staining of K562 cells in which the expression of both FLVCR1a and FLVCR1b, or FLVCR1a alone, was downregulated using specific shRNA, compared with control cells. Erythroid differentiation was induced with sodium butyrate ( $0.5 \mathrm{mM}, 72$ hours). $n=6$. Two-way ANOVA. (F) Benzidine staining of silenced K562 cells stimulated with hemin ( $5 \mu \mathrm{M} ; 72$ hours). The percentage of benzidine-positive cells is indicated. Values represent mean \pm SEM. $n=6$. Two-way ANOVA. ${ }^{*} P<0.05 ;{ }^{* *} P<0.01 ;{ }^{* * *} P<0.001$.

was determined in these cells. The quantity of cytochrome $c$ oxidase was unaltered (Figure 2H) in HeLa cells depleted for FLV$\mathrm{CR} 1 \mathrm{a}$ and FLVCR11b compared with controls. These data indicate that Flvcr1b silencing results in an increased free-unbound heme in mitochondria. Taken together, these data demonstrate that FLVCR1b is able to regulate heme efflux from the mitochondrion.

To assess whether mitochondrial heme accumulation due to the loss of FLVCR1b affects mitochondrial functionality, the mitochondrial $\mathrm{Ca}^{2+}$ response after agonist stimulation was monitored as a highly sensitive readout of mitochondrial state. It is well known that mitochondrial alterations cause defects in $\mathrm{Ca}^{2+}$ uptake by the organelle (15). The dynamics of agonist-dependent
$\mathrm{Ca}^{2+}$ concentration changes were measured taking advantages of a mitochondria-targeted chimera of the $\mathrm{Ca}^{2+}$-sensitive photoprotein aequorin (16). We investigated the $\mathrm{Ca}^{2+}$ response to histamine, which acts on $\mathrm{Gq}$-coupled plasma membrane receptors and causes the production of inositol 1,4,5 trisphosphate (InsP3), thus inducing the release of $\mathrm{Ca}^{2+}$ from the ER to mitochondria (17). The silencing of FLVCR1a and FLVCR1b in HeLa cells caused a significant reduction of $\mathrm{Ca}^{2+}$ spike in the mitochondrial matrix evoked by agonist stimulation corresponding to a $31 \%$ decrease in amplitude versus control cells (Figure 2I). These data suggest that in the absence of FLVCR1b, heme accumulates in the mitochondria, resulting in the alterations of mitochondrial functionality. 
A
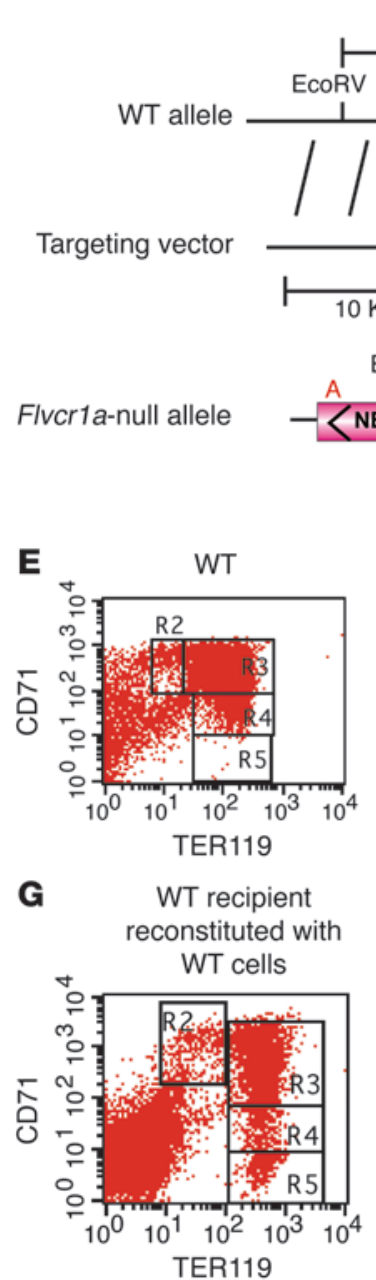

B

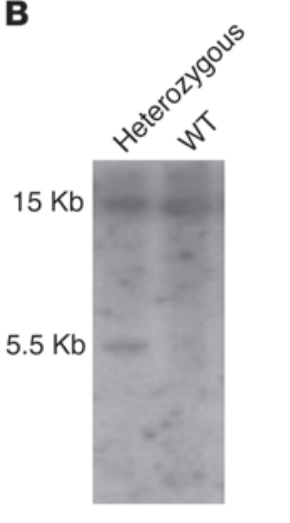

F

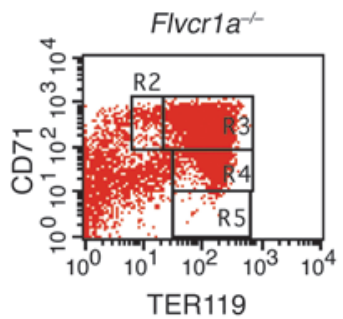

WT recipient reconstituted with Flvcr1a $a^{--}$cells

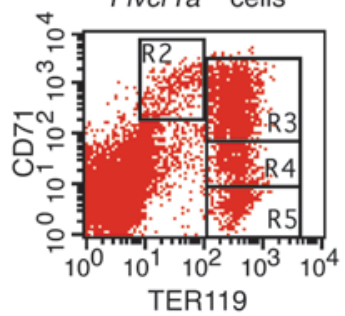

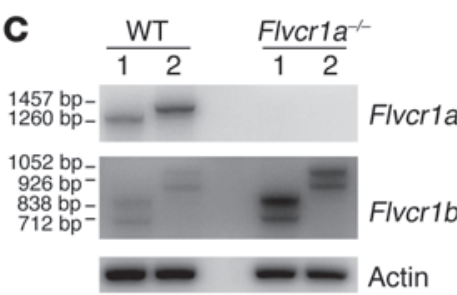

D

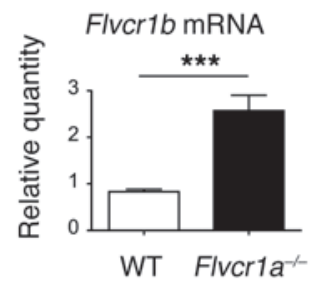

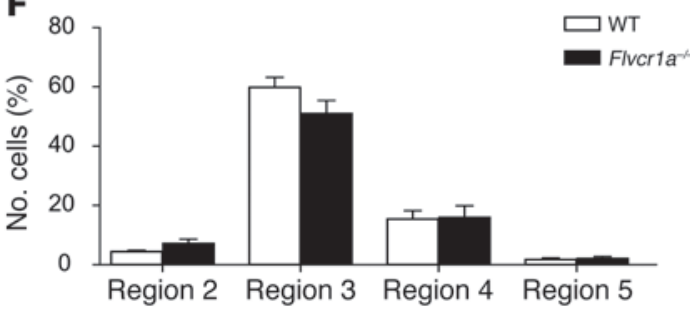

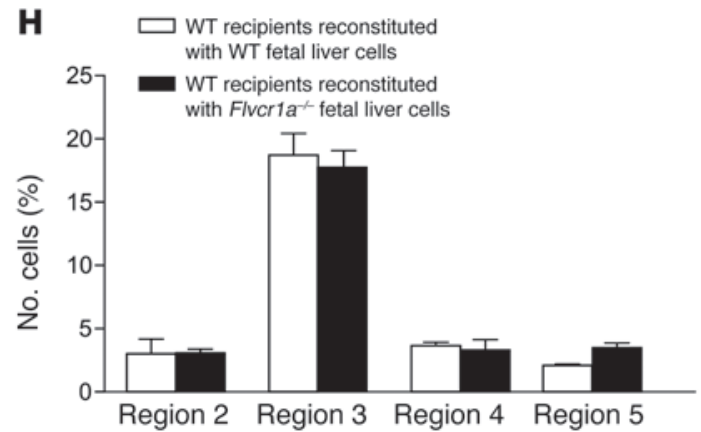

Figure 4

FLVCR1b controls erythroid differentiation in vivo. (A) Schematic representation of the targeting strategy. The start codon in the first exon of the Flvcr1 gene was disrupted by the insertion of the neomycin resistance cassette by homologous recombination in mouse ES cells. (B) Southern blot analysis on genomic DNA extracted from a recombinant and a wild-type clone, digested with EcoRV, and hybridized with the probe shown in A. (C) RT-PCR analysis of Flvcr1 isoforms levels in wild-type and Flvcr1 $a^{-/}$embryos at E13.5. Two different pairs of primers were used in lanes 1 and 2. (D) qRT-PCR analysis of Flvcr1b mRNA on E13.5 wild-type and Flvcr1a ${ }^{-/-}$embryos. $n=6 . t$ test. ${ }^{* \star *} P<0.001$. (E) Erythroid differentiation is completely normal in Flvcr1 $a^{-/-}$fetal liver. Representative flow cytometric analyses of E14.5 liver cells from wild-type and Flvcr1a ${ }^{-/-}$embryos immunostained with antibodies to CD71 and Ter119. Regions R2-R5 corresponding to different maturational stages are indicated. The percentage of cells in each population is reported $(\mathbf{F}) . n=6$. (G) Representative flow cytometric analyses of bone marrow cells, isolated from mice transplanted with wild-type or Flvcr1 $a^{-/}$fetal liver cells, immunostained with antibodies to CD71 and Ter119. Regions R2-R5 corresponding to different maturational stages are indicated. The percentage of cells in each population is reported $(\mathbf{H}) . n=6$. Values represent mean $\pm \mathrm{SEM}$.

FLVCR1b is essential for erythroid differentiation in vitro. It is well known that intracellular heme content in erythroid progenitors controls differentiation by the activation of the transcription and translation of globin chains $(1,2)$. As FLVCR1b regulates heme efflux from the mitochondrion, it could likely be involved in the hemoglobinization of erythroid precursors. This hypothesis was supported by the observation that FLVCR1b expression is regulated following the stimulation of erythropoiesis. Indeed, $\mathrm{K} 562$ cells were induced to differentiate in vitro by the treatment with the heme precursor ALA, which directly stimulates heme synthesis. As a control, heme concentration and globin mRNA levels were checked and were found to be increased already 24 hours after the treatment (Figure 3A). In these conditions, the Flvcr $1 b$ transcript was strongly upregulated. The increase of Flvcr1b mRNA levels positively correlated with the increased expression of Alas2, the rate-limiting enzyme in the heme biosynthetic pathway (Figure 3A). The same result was obtained following the stimulation of K562 cell differentiation with sodium butyrate (D. Chiabrando, unpublished observations) and in vivo following the stimulation of erythropoiesis by phlebotomy (Figure 3B).

To address the role of FLVCR1b and $1 \mathrm{a}$ in erythropoiesis, overexpression and silencing experiments in K562 cells were performed. 


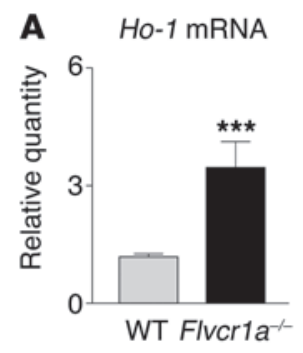

D

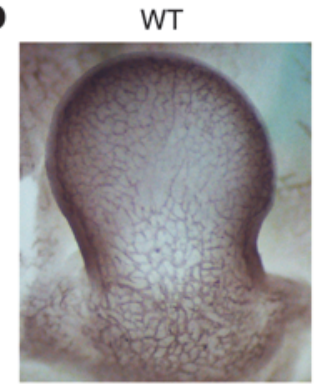

B

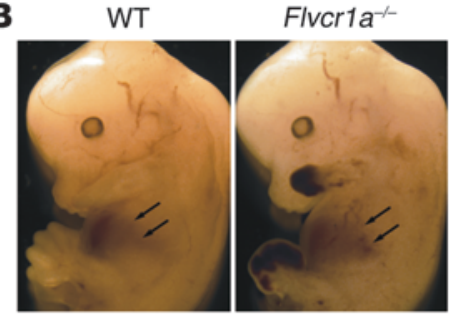

E12.5

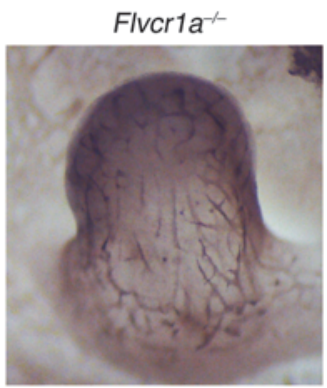

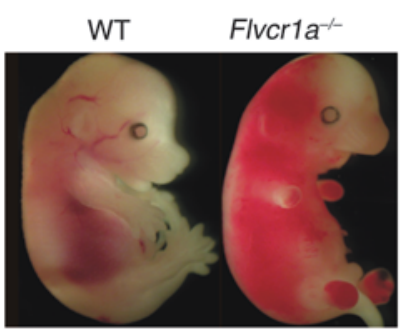

E15.5

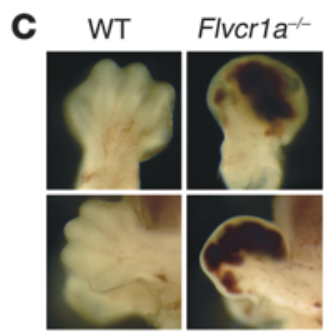

E12.5

E
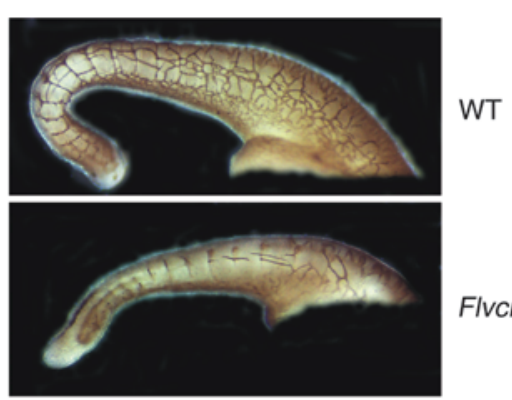

Flvcr1a $^{-1-}$

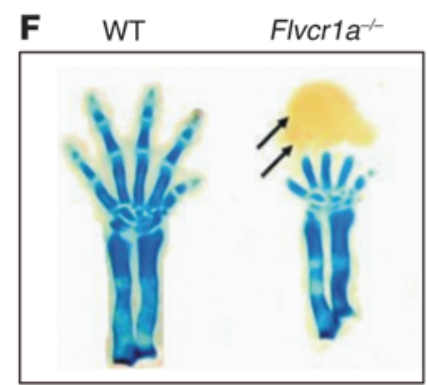

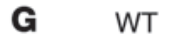

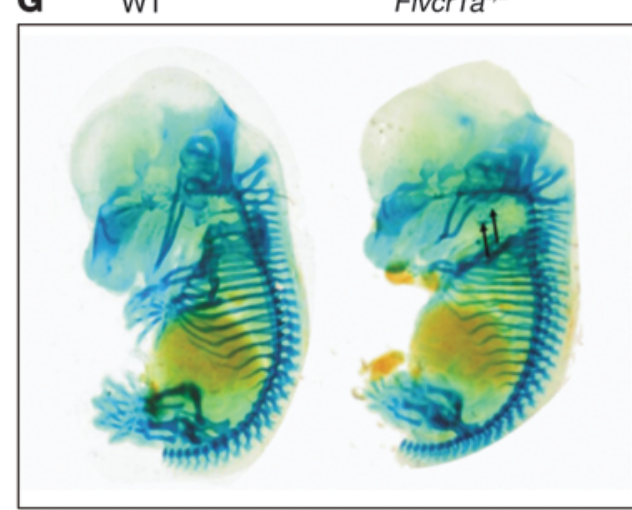

H WT

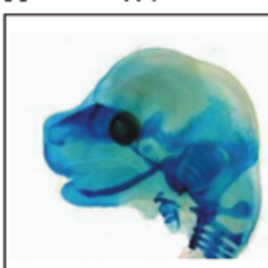

Flvcr1a $^{-1}$

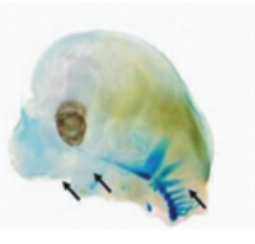

\section{Figure 5}

Flvcr1 ${ }^{-/-}$embryos show hemorrhages, edema, and skeletal malformations. (A) qRT-PCR analysis showing the upregulation of Ho-1 mRNA in Flvcr1 $a^{-/-}$embryos. Values represent mean \pm SEM. $n=6$. $t$ test. ${ }^{* \star} P<0.001$. (B) Multifocal and extended hemorrhages and edema in E12.5 and E15.5 Flvcr1a ${ }^{-/-}$embryos. (C) Enlarged view of E12.5 wild-type and F/vcr1 a ${ }^{-/-}$limbs. (D and E) Whole-mount immunohistochemical analysis of E11.5 wild-type and Flvcr1 $a^{-/-}$embryos using anti-PECAM antibody showing abnormal vasculogenesis in Flvcr1a knockout animal. An enlarged view of the anterior limb (D) and tail $(\mathbf{E})$ is shown. $(\mathbf{F}-\mathbf{H})$ Skeletal malformations in E14.5 Flvcr1 $\mathrm{a}^{-/-}$embryos were analyzed by alcian blue/alizarin red staining. (F) An enlarged view of the anterior limb of E14.5 embryos: digits do not form properly in the Flvcr1a ${ }^{-/-}$embryo. Different grades of skeletal malformations are shown: Meckel's cartilage (arrows) is reduced in the Flvcr1 $a^{-/-}$embryo (G); head cartilages are severely compromised and the lower jaw (arrows) completely absent in the Flvcr1a ${ }^{-/-}$embryo (H).

As observed in HeLa cells, the overexpression of FLVCR1a in K562 cells resulted in a slight but significant decrease of heme content, whereas the overexpression of FLVCR1b caused an increase of heme levels that was prevented by the treatment with succinylacetone (Supplemental Figure 7). In agreement with heme content, increased expression of globin mRNA (Figure 3C) and a higher percentage of benzidine-positive cells (Figure 3D) were observed in K562 cells following the overexpression of FLVCR1b compared with control cells. On the contrary, FLVCR1a-overexpressing K562 cells were not able to differentiate (Figure 3, C and D). Therefore, the overexpression of FLVCR1b promotes in vitro erythroid differentiation by increasing heme biosynthesis rate.

Interestingly, shRNA-mediated downregulation of FLVCR1a (Supplemental Figure 5) led to the elevation of the percentage of benzidine-positive cells compared with controls, which further increased upon the stimulation of erythroid differentiation using sodium butyrate (Figure $3 \mathrm{E}$ ). Thus, the loss of the membrane heme exporter FLVCR1a promotes in vitro erythroid differentiation, likely by increasing total intracellular heme levels.

A decrease in the percentage of benzidine-positive cells was observed in K562 cells lacking both FLVCR1 isoforms compared with FLVCR1a-deficient cells (Figure 3E). Moreover these cells were not able to differentiate upon stimulation with sodium butyrate (Figure 3E). Thus, FLVCR1b, by controlling heme efflux from the mitochondrion, is fundamental for proper hemoglobinization of K562 cells. The direct administration of hemin, which bypasses the heme biosynthesis step, completely rescues the defect of FLVCR1aand FLVCR1b-deficient K562 cells (Figure 3F). 


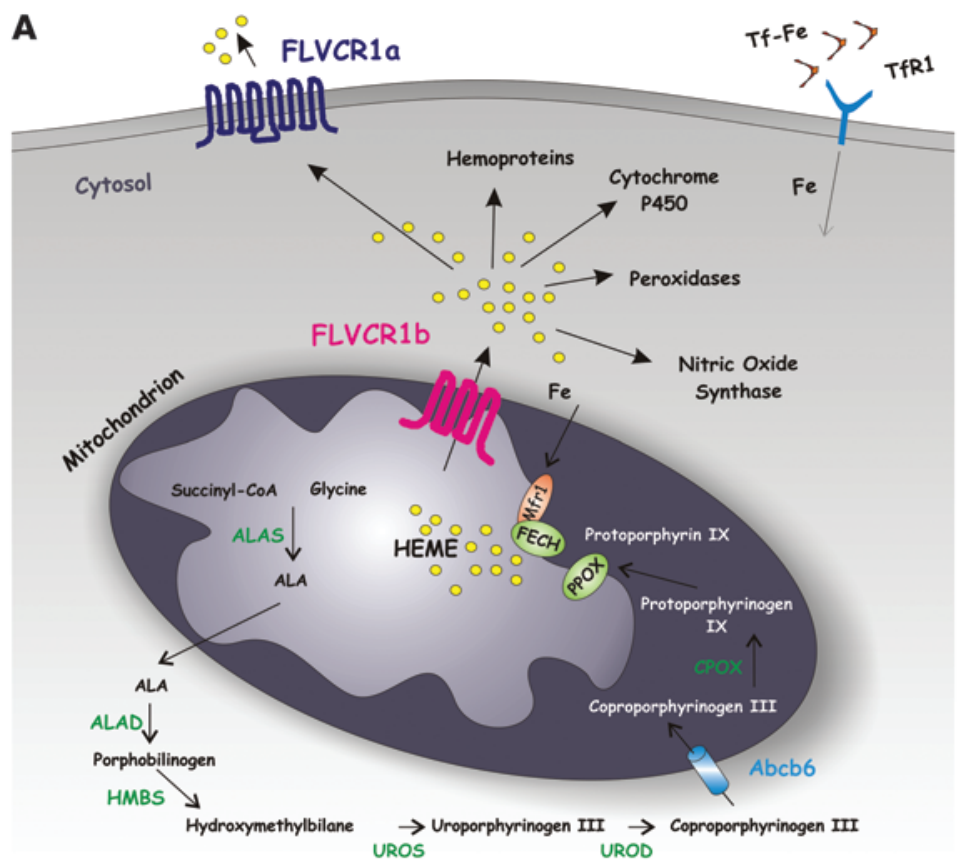

B

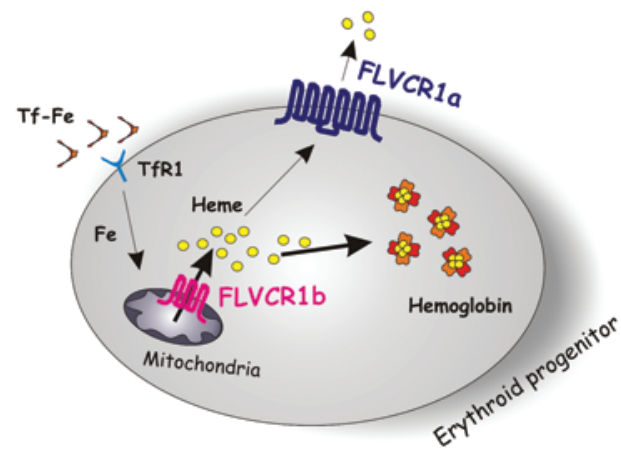

Figure 6

A model for FLVCR1 isoforms function. (A) A schematic representation of heme biosynthesis is shown (see text for details). Transferrin-bound iron (Tf-Fe) is taken up by cells through transferrin receptor 1 (TfR1), and iron is transferred to the mitochondrion for heme biosynthesis. It was reported that the mitochondrial iron importer MITOFERRIN1 (MFRN1) and FECH are part of the same complex in the inner mitochondrial membrane. FLVCR1b could work in close association with this complex to allow heme export out of the mitochondrion for incorporation into new hemoproteins. Heme not used for hemoprotein synthesis is exported out of the cell through the cell-surface isoform FLVCR1a. (B) During erythroid differentiation, the expression of FLVCR1b in the mitochondrion regulates heme export into the cytosol, allowing hemoglobinization of erythroid precursors. At the cell membrane, FLVCR1a regulates the export of heme in excess. The data reported here suggest that decreased expression of the membrane heme exporter FLVCR1a and increased expression of FLVCR1b are fundamental for proper differentiation of erythroid progenitors.

FLVCR1b is essential for erythroid differentiation in vivo. It has already been reported that Flvcr1-null mice die in utero due to a complete block of erythroid differentiation at the proerythroblast stage (4). In that work, the inactivation of the Flvor1 gene was obtained by deleting the third exon, thus likely affecting the expression of both Flvcr1a and Flvcr1b. Based on our results on K562 cells, we hypothesized that the erythroid defect in Flvor1-null mice (4) was due to the lack of Flvor1b rather than to Flvcrla deficiency. To test this hypothesis we analyzed eryth- ropoiesis in mice lacking Flvcr1a, but still expressing FLVCR1b (Flvcr1a ${ }^{-/-}$mice).

The specific loss of Flvcrla isoform was achieved by the insertion of the neomycin resistance cassette in the first exon of the Flver1 gene, thus disrupting its start codon (Figure 4, A and B). As expected, Flvcr1 $a^{-/}$embryos did not express Flvcr1a mRNA (Figure 4C), while Flvcr1b transcript was upregulated (Figure 4, C and D). Flvcrla-/embryos died during embryonic development between E14.5 and birth (Supplemental Table 1). Rarely, some dead Flvcr1a $a^{-/}$newborns were found, whereas no Flvcr1a ${ }^{-/-}$ animal was found at weaning.

Definitive erythropoiesis was analyzed by flow cytometry on E14.5 fetal liver cells double-stained for Ter119 (erythroid-specific antigen) and CD71 (transferrin receptor). Normal erythroid differentiation was observed in Flvcrla ${ }^{-/-}$embryos (Figure 4, E and F), indicating that FLVCR1b alone is sufficient to support fetal erythropoiesis when the expression of FLVCR1a is lost. In addition, we tested E14.5 Flvcrla $a^{-/}$fetal liver cell repopulation capacity. Notably, Flvcr1 $a^{-/-}$cells were able to fully reconstitute erythropoiesis when transplanted into lethally irradiated wild-type recipients. Erythroid differentiation was comparable between recipient mice transplanted with wild-type or Flvcr1 $1 a^{-/-}$fetal liver cells (Figure 4, G and H). Together these data indicate that FLVCR1b, rather than FLVCR1a, is absolutely needed to allow erythroid differentiation.

Loss of FLVCR1a causes vascular and skeletal defects in mice. As shown in Figure 4, C and D, Flvcrla ablation in mice was associated with the upregulation of Flvcr1b expression. Based on our data on HeLa cells showing that FLVCR1b overexpression promotes heme synthesis, we hypothesized that Flvcr1 $a^{-/-}$embryos experienced a condition of heme overload further exacerbated by FLVCR1a deficiency. Consistently, a strong induction of $\mathrm{Ho}-1 \mathrm{mRNA}$, which is directly regulated by heme level, was observed in Flvcr1a $a^{-/}$embryos (Figure 5A).

As mentioned before, Flvcrla $a^{-/-}$embryos died between E14.5 and birth (Supplemental Table 1). The embryonic death of Flvcrla $a^{-1}$ mice was likely due to multifocal and extended hemorrhages, evident in the primordial limbs at E12.5 and then throughout the head and body, associated with subcutaneous edema (Figure 5, B and C). To investigate the overall vascular architecture, whole-mount PECAM1 immunostaining was performed on E11.5 Flvcr1 $1 a^{-/-}$embryos. Flvcrla $a^{-/}$embryos showed reduced vasculature extension and complexity compared with controls. This was particularly evident in the primordial limbs and tail, where vessels did not form properly and branching was severely compromised (Figure 5, D and E). In addition to vascular defects, Flvcr1 $a^{-/-}$embryos showed skeletal abnormalities similar to those reported in mice lacking both Flvcr1 isoforms (4). Skeletal malformations were evident in the primordial limbs where digital cartilages were shorter and in the head where the nasal and Meckel's cartilage were incomplete (Figure 5, F-H). These results indicate that FLVCR1a expression is important to prevent endothelial ruptures related to hemorrhages, edema, and consequent malformations, thus highlighting a still unappreciated role for FLVCR1 in these processes. 


\section{Discussion}

Heme is endogenously synthesized in the mitochondria. The existence of a mitochondrial heme exporter has therefore been hypothesized, as an energy-dependent mechanism is required for heme to cross the 2 mitochondrial membranes (18). However, a mitochondrial heme exporter has not been identified to date.

In this work, we report the identification of Flver $1 b$ as a mitochondrial isoform of the heme exporter Flvcr1. FLVCR1b is a member of the major facilitator superfamily (MFS) and has 6 hydrophobic transmembrane domains. It is likely that FLVCR1b homodimerizes to form a transporter with 12 transmembrane domains similar to FLVCR1a. It has been previously reported that other members of the MFS with 6 transmembrane domains are able to homodimerize $(19,20)$. Several lines of evidence suggest that FLVCR1b is a mitochondrial heme exporter. The overexpression of FLVCR1b in vitro results in heme accumulation in the cytosol. In addition, FLVCR1b overexpression in Flvcr1 $a^{-/-}$embryos causes an increase in heme concentration, as suggested by the upregulation of Ho-1 mRNA. On the other hand, mitochondrial heme overload was observed in FLVCR1b-silenced HeLa cells compared with controls, demonstrating that FLVCR1b plays a role in mitochondrial heme efflux. The mitochondrial heme overload caused by FLVCR1b silencing was associated with a deregulation of mitochondrial $\mathrm{Ca}^{2+}$ homeostasis, suggesting a toxic effect of heme overload in the mitochondrial matrix.

The molecular mechanisms through which heme is exported out of the mitochondrion are still unclear (21). FECH, the last enzyme in the heme biosynthetic pathway, which catalyzes the insertion of iron into PPIX, forms an oligomeric complex on the inner mitochondrial membrane that include ABCB10 and MITOFERRIN1 (MFRN1). The association of $\mathrm{FECH}$ with the mitochondrial iron importer MFRN1 allows the direct transfer of iron to $\mathrm{FECH}$, facilitating heme biosynthesis (22), while ABCB10 plays an important role in the stabilization of MFRN1 expression (23). One intriguing hypothesis is that the mitochondrial heme exporter FLVCR1b represents an additional component of this complex, allowing the direct transfer of heme out of the mitochondrion soon after its synthesis (Figure 6A).

It has been previously demonstrated that the loss of both Flver 1 isoforms in mice leads to embryonic lethality due to an impairment of erythroid differentiation at the proerythroblast stage (4). Here, we show that erythropoiesis is completely normal in mice only lacking Flvcrla but overexpressing Flvcr1b, highlighting a fundamental role for Flvcr1b during erythroid differentiation. Transplantation of Flvcr1 $a^{-/}$fetal liver cells into lethally irradiated wildtype recipients fully confirms this hypothesis. Taken together, these data highlight a fundamental role for FLVCR1b during erythroid differentiation. The comparison between Flvcrla-/- embryos and the previously reported mouse model of Flvcr1 deficiency (4) suggests that the block of erythroid differentiation evident in Flvcrla/b-null embryos (4) is likely to be due to an impairment of heme biosynthesis caused by the loss of FLVCR1b rather than to the accumulation of heme caused by FLVCR1a deficiency. This conclusion is further supported by our in vitro data showing that the depletion of FLVCR1a using shRNA promotes erythroid differentiation, while the loss of both forms of FLVCR1 isoforms blocks in vitro differentiation. In agreement with this conclusion is the observation that mutations in the first exon of the Flvorl gene, which likely only affect the activity of the FLVCR1a isoform, have been described in patients affected with posterior column ataxia and retinitis pigmentosa (PCARP) who are not anemic $(24,25)$.
This suggests that the impairment of FLVCR1a alone is not sufficient to block erythropoiesis and further supports the idea that FLVCR1b is a major player during erythropoiesis.

Feline leukemia virus subgroup C (FeLV-C) is thought to cause anemia in cats because, by binding to the FLVCR1 receptor, it blocks FLVCR1 function at the cell membrane and, once inside the cell, it binds to FLVCR1 in the ER or Golgi apparatus, thus impairing its expression at the cell surface $(3,10,11,26)$. A similar mechanism has been proposed to explain why FeLV-C infection of K562 cells impairs their ability to undergo erythroid differentiation (3). Based on structural similarity between FLVCR1a and FLVCR1b proteins, we hypothesized that FeLV-C envelope protein could also bind to FLVCR1b protein in the ER soon after its synthesis, thus preventing its expression in the mitochondrion. According to our hypothesis, some FLVCR1 regions critical for FeLV-C envelope protein binding are located in portions of the protein common to FLVCR1a and FLVCR1b (27).

Flvcr1a $a^{-1-}$ embryos develop hemorrhages, edema, and skeletal malformations. Similar phenotypes, displaying hemorrhages and edema, have also been demonstrated in mouse models with alteration of endothelial integrity (28), suggesting that endothelial damage could lead to the observed phenotype. Accordingly, incomplete vasculogenesis occurs in Flvcrla $a^{-/}$embryos prior to the onset of hemorrhages. Our data clearly indicate that, since E11.5 alterations of vascular arborization were evident in the limbs. A similar phenotype of hemorrhages associated with reduced vasculature complexity was reported in another mouse model (29).

Endothelial cells are highly sensitive to heme overload. Therefore, we could hypothesize that in Flvcrla $a^{-1-}$ endothelial cells, the lack of a cell membrane heme exporter, together with enhanced heme synthesis promoted by increased FLVCR1b expression, could lead to intracellular heme overload and finally to oxidative stress that could account for the observed phenotypes. These data suggest that in endothelial cells, such as in erythroid progenitors, the coordinated expression of FLVCR1 isoforms is crucial to maintaining heme homeostasis.

Flvcr $1 a^{-/-}$embryos also showed skeletal malformations. It is well known that tissue hypoxia impairs proper cartilage development (30). Therefore, it is likely that the impairment of endothelial integrity observed when the expression of FLVCR1a is lost could finally lead to tissue hypoxia and consequent malformations. Skeletal abnormalities have also been reported in Flvcr1a/b-null mice (4), where hypoxia is further exacerbated by the failure of erythropoiesis.

In conclusion, our data demonstrate that tissues highly sensitive to heme are also highly susceptible to altered expression of Flvcr1 isoforms. Particularly, for erythroid progenitors, we hypothesize that the 2 Flver 1 isoforms are involved in distinct steps during erythroid differentiation. According to the model illustrated in Figure 6B, the expression of FLVCR1b in mitochondria of differentiating erythroid progenitors allows heme biosynthesis and heme efflux into the cytosol, a process essential for hemoglobinization and erythroid differentiation. Loss of FLVCR1b, as occurs in Flvcr1a/b-null mice (4), leads to a block of differentiation. Decreased expression of the cell membrane heme exporter FLVCR1a also contributes to the erythroid differentiation process by limiting the excretion of heme through the cell membrane, as previously shown (3). Therefore, the coordinated regulation of the 2 Flver 1 isoforms is required for a complete in vivo differentiation of erythroid progenitors. Misregulation of Flvcr1 isoform expression could contribute to the pathogenesis of diseases characterized by abnormal heme levels. 


\section{Methods}

Cell culture. The human epithelial HeLa cell line (ATCC no. CCL-2) and the human lymphoblast K562 cell line (ATCC no. CCL-243) were propagated in RPMI medium (Invitrogen). The rat renal epithelial NRK-52E cell line (ATCC no. CRL-1571) and the human embryonic kidney HEK293 cell line (ATCC no. CRL-1573) were propagated in DMEM (Invitrogen). All the media were supplemented with $10 \%$ heat-inactivated low-endotoxin fetal bovine serum (Invitrogen), $100 \mathrm{U} / \mathrm{ml}$ penicillin, and $100 \mu \mathrm{g} / \mathrm{ml}$ streptomycin. Cells were maintained at $37^{\circ} \mathrm{C}$ under a $5 \% \mathrm{CO}_{2}$ atmosphere.

For in vitro differentiation studies, K562 cells were stimulated with $0.5 \mathrm{M}$ sodium butyrate (SB; 303410; Sigma-Aldrich) or $50 \mu \mathrm{M}$ Hemin (H651-9; Frontier Scientific), as previously described (3). To specifically activate or inhibit heme biosynthesis, HeLa and K562 cell lines were treated with $5 \mathrm{mM}$ ALA (A3785; Sigma-Aldrich) or $1 \mathrm{mM}$ succinylacetone (4,6-dioxoheptanoic acid; D1415; Sigma-Aldrich) respectively.

Benzidine staining. To evaluate in vitro erythroid differentiation, percentage of benzidine-positive cells was determined according to standard protocols (31).

Subcellular fractionation. $10^{9}$ Cells were harvested, washed in phosphatebuffered saline medium, pelleted by centrifugation at $500 \mathrm{~g}$ for 5 minutes, resuspended in homogenization buffer $(0.25 \mathrm{M}$ sucrose and $10 \mathrm{mM}$ Hepes $\mathrm{pH}$ 7.4) and gently disrupted by dounce homogenization. The homogenate was centrifuged twice at $600 \mathrm{~g}$ for 5 minutes to remove cellular debris and nuclei, and the supernatant was centrifuged at $10.300 \mathrm{~g}$ for 10 minutes to pellet crude mitochondria. The resultant supernatant was centrifuged at $100,000 \mathrm{~g}$ for 1 hour in a Beckman 70 Ti rotor at $4^{\circ} \mathrm{C}$ to pellet microsomes, which were resuspended in homogenization buffer. The mitochondrial pellet, resuspended in isolation medium $(250 \mathrm{mM}$ mannitol, $5 \mathrm{mM}$ Hepes [pH 7.4], and $0.5 \mathrm{mM}$ EGTA), was layered on top of $8 \mathrm{ml}$ of Percoll medium (225 mM mannitol, $25 \mathrm{mM}$ Hepes [pH 7.4], 1 mM EGTA, and 30\% Percoll $[\mathrm{v} / \mathrm{v}])$ in a $10-\mathrm{ml}$ polycarbonate ultracentrifuge tube and centrifuged for 30 minutes at $95,000 \mathrm{~g}$. A dense band containing purified mitochondria, recovered approximately three-quarters down the tube, was removed, diluted with isolation medium, washed twice by centrifugation at $6,300 \mathrm{~g}$ for 10 minutes to remove the Percoll, and finally resuspended in isolation medium. MAM, removed from the Percoll gradient as a diffuse white band located above the mitochondria, were diluted in isolation medium and centrifuged at 6,300 $\mathrm{g}$ for 10 minutes. The supernatant containing MAM was centrifuged at $100,000 \mathrm{~g}$ for 1 hour in a Beckman 70 Ti rotor, and the resulting pellet was resuspended in the homogenization buffer (13).

Western blot. For Western blot analysis, $50 \mu \mathrm{g}$ of protein extracts were loaded on $10 \%$ or $15 \%$ SDS-polyacrylamide gels and analyzed by Western blotting. The following antibodies were used: anti-myc antibody (32), anti-GAPDH antibody (MAB374; Millipore), anti-HADHA antibody (ab54477; Abcam), anti-IP3R3 (610312; BD), anti-panVDAC (ab15895; Abcam), anti- $\beta$-tubulin (T5201; Sigma-Aldrich), antiSigmaR1 (HPA018002; Sigma-Aldrich), and anti-human FLVCR1 antibody (NB100-1481; Novus Biologicals).

Measurements complex IV quantity. A commercial multiplexing microplate kit (Abcam 109910) was used to determine the quantity of cytochrome $c$ oxidase. The COX enzyme was immunocaptured within the wells of the microplate, and the quantity of the enzyme was determined by adding a COX-specific detector antibody and the appropriate alkaline phosphataseconjugated antibody; this phosphatase changes a substrate from colorless to yellow at $405 \mathrm{~nm}$. The rate of reaction was proportional to the amount of protein captured in the wells.

Aequorin measurements. Cells grown on $13-\mathrm{mm}$ round glass coverslips at $50 \%$ confluence were transfected with mitochondrial-targeted aequorin (mtAEQ).

All aequorin measurements were carried out in KRB (125 mM NaCl, $5 \mathrm{mM}$ $\mathrm{KCl}, 1 \mathrm{mM} \mathrm{MgSO}$, $1 \mathrm{mM} \mathrm{Na}_{2} \mathrm{HPO}_{4}, 5.5 \mathrm{mM}$ glucose, $20 \mathrm{mM} \mathrm{NaHCO}_{3}$,
$2 \mathrm{mM}$ L-glutamine and $20 \mathrm{mM}$ HEPES, $\mathrm{pH}$ 7.4, and was supplemented with $1 \mathrm{mM} \mathrm{CaCl}_{2}$ ). Histamine was added to the same medium. The experiments were terminated by lysing the cells with $100 \mu \mathrm{M}$ digitonin in a hypotonic $\mathrm{Ca}^{2+}$-rich solution $\left(10 \mathrm{mM} \mathrm{CaCl}_{2}\right.$ in $\mathrm{H}_{2} \mathrm{O}$ ), thus discharging the remaining aequorin pool. The light signal was collected and calibrated into $\left[\mathrm{Ca}^{2+}\right]$ values, as previously described (16).

Phlebotomy. Phlebotomy was performed according to standard methods (33). RNA extraction and real-time PCR analysis. Total RNA extraction and realtime PCR were performed as previously reported (33). To discriminate between Flvcr1a and Flvcr1b, specific primers and probes were designed using Primer Express Software version 3.0 (Applied Biosystems); see Supplemental Table 2 for details.

Measurement of heme concentration. Intracellular heme concentration was measured using a fluorescence assay, as previously reported (34). Briefly, cells were collected and resuspended in $2 \mathrm{M}$ oxalic acid and heated at $100^{\circ} \mathrm{C}$ for 30 minutes, leading to iron removal from heme. The resultant protoporphyrin was measured by fluorescence ( $400 \mathrm{~nm}$ excitation and 662 or $608 \mathrm{~nm}$ emission). Data were normalized to the endogenous protoporphyrin content (by measuring the fluorescence of not-heated samples) and to total protein concentration. Results were expressed as $\mathrm{pmol}$ of heme/mg total protein. The same procedure was used on crude mitochondrial and cytosolic fractions.

Generation of Flvcr1a-1- embryos. A neomycin-resistance cassette was cloned into the ATG start codon in the first exon of Flvcr1, and this construct was transfected by electroporation in mouse ES cells. Three clones carrying the targeted allele were identified by Southern blotting and 2 of them injected into C57BL/ 6 mouse blastocysts. Chimeras were used to obtain heterozygous mice carrying the Flvcrlat-/ allele. Both inbred 129Sv and mixed 129Sv $\times$ C57BL/6 Flvcr1a heterozygous mice (Flvor $\left.1 a^{+/-}\right)$were generated.

Transplantation assay. Fetal liver cells were obtained from E14.5 wild-type and Flvcr $1 a^{-/-}$embryos. From 1 to $3 \times 10^{6}$ cells were injected into the tail vein of lethally irradiated wild-type mice. Transplanted mice were analyzed after 4 weeks of recovery. The transplantation rate was assessed on blood genomic DNA by PCR using specific primers to amplify the wild-type or the knockout allele (Supplemental Figure 8).

Alcian blue staining. Cartilage morphology was analyzed by alcian blue/ alizarin red staining on E14.5 and E15.5 embryos, according to previously described methods (35).

Whole-mount immunostaining. Whole-mount staining was performed as described (35), using anti-mouse CD31 (Pecam-1) monoclonal antibody (BD Biosciences). DAB/hydrogen peroxide was used for visualization.

Analysis of erythroid differentiation. Erythroid differentiation was analyzed as previously reported $(4,36)$. Briefly, single-cell suspensions were prepared from freshly isolated E14.5 fetal liver cells and were immunostained with anti-TER119-PE and anti-CD71-FITC (BD Biosciences - Pharmingen), then subjected to flow cytometry.

Flver1 cloning and lentiviral infection. The lentiviruses pCCL.ET.GFP.sin, pCCL.ET.Flvcr1a-myc, pCCL.ET.Flvcr1b-myc, which express GFP, murine Flvcrla-myc, or murine Flvcr1b-myc under the control of the enhanced transthyretin promoter, were produced in HEK293FT cells as previously described (37-39). HeLa and K562 cells were infected with the lentiviruses in the presence of Sequa-brene. To obtain FLVCR1b overexpression, cells were infected twice in a week. Expression of FLVCR1b protein was checked 24 hours following lentiviral infection, while overexpression studies were performed 72 hours after infection. Cells were infected once for FLVCR1a overexpression.

FLVCR1 silencing. A set of 5 clones (RHS4533-NM_014053) targeting human Flvcr1 gene were purchased (Open Biosystem). A shRNA against the first exon of human Flvcrl gene was used to specifically downregulate 
FLVCR1a expression; another one was used against exon 9, to abrogate both Flvcr1a and Flvcr1b expression. Following lentiviral infection, cells were selected with $0.02 \mu \mathrm{g} / \mathrm{ml}$ puromycin.

To specifically target FLVCR1b expression, an siRNA against the 5' UTR of the Flvcr1b mRNA was designed (TCTGTTAATTGCCAGCATT). Scrambled siRNA was obtained from Darmacon. FLVCR1b and scrambled siRNA were transiently transfected in HeLa cells using Lipofectamine 2000 (Invitrogen).

Statistics. Results were expressed as mean \pm SEM. Statistical analyses were performed using 1-way or 2-way ANOVA or Student's $t$ test. A $P$ value of less than 0.05 was considered significant.

Study approval. Animal studies were approved by the animal ethical committee of the University of Torino, Torino, Italy.

\section{Acknowledgments}

For technical help, we thank E. De Luca for confocal microscopy and E. Messana for generation of knockout mice. We thank E. Hirsch and P.P. Pandolfi for critical discussion and reading the manuscript. We are grateful to Radhika Srinivasan for editing the manuscript. This work was supported by the Diamond Blackfan Anemia Foundation (to E. Tolosano), Regione Piemonte (to E. Tolosano and F. Altruda), the Italian Association for Cancer Research (AIRC), Telethon (GGP09128 and GGP11139B), local funds from the University of Ferrara, the Italian Ministry of Education, University, and Research (COFIN, FIRB and Futuro in Ricerca), the Italian Cystic Fibrosis Research Foundation, and the Italian Ministry of Health (to P. Pinton).

Received for publication December 13, 2011, and accepted in revised form September 27, 2012.

Address correspondence to: Emanuela Tolosano, Molecular Biotechnology Center, Department of Genetics, Biology, and Biochemistry, Via Nizza 52, 10126 Torino, Italy. Phone: 39.011.6706423; Fax:39.011.6706432; E-mail: emanuela.tolosano@ unito.it.
1. Chen JJ. Regulation of protein synthesis by the heme-regulated eIF2alpha kinase: relevance to anemias. Blood. 2007;109(7):2693-2699.

2. Furuyama K, Kaneko K, Vargas PD. Heme as a magnificent molecule with multiple missions: heme determines its own fate and governs cellular homeostasis. Tohoku J Exp Med. 2007;213(1):1-16.

3. Quigley JG, et al. Identification of a human heme exporter that is essential for erythropoiesis. Cell.2004; 118(6):757-766.

4. Keel SB, et al. A heme export protein is required for red blood cell differentiation and iron homeostasis. Science. 2008;319(5864):825-828.

5. Ponka P. Cell biology of heme. Am J Med Sci. 1999; 318(4):241-256.

6. Ajioka RS, Phillips JD, Kushner JP. Biosynthesis of heme in mammals. Biochim Biophys Acta. 2006; 1763(7):723-736.

7. Khan AA, Quigley JG. Control of intracellular heme levels: heme transporters and heme oxygenases. Biochim Biophys Acta. 2011;1813(5):668-682.

8. Schultz IJ, Chen C, Paw BH, Hamza I. Iron and porphyrin trafficking in heme biogenesis. J Biol Chem. 2010;285(35):26753-26759.

9. Krishnamurthy PC, et al. Identification of a mammalian mitochondrial porphyrin transporter. Nature. 2006;443(7111):586-589.

10. Quigley JG, et al. Cloning of the cellular receptor for feline leukemia virus subgroup C (FeLV-C), a retrovirus that induces red cell aplasia. Blood. 2000; 95(3):1093-1099.

11. Tailor CS, Willett BJ, Kabat D. A putative cell surface receptor for anemia-inducing feline leukemia virus subgroup $C$ is a member of a transporter superfamily. J Virol. 1999;73(8):6500-6505.

12. Claros MG, Vincens P. Computational method to predict mitochondrially imported proteins and their targeting sequences. Eur J Biochem. 1996; 241(3):779-786.

13. Wieckowski MR, Giorgi C, Lebiedzinska M, Duszynski J, Pinton P. Isolation of mitochondria-associated membranes and mitochondria from animal tissues and cells. Nat Protoc. 2009;4(11):1582-1590.

14. Ryter SW, Tyrrell RM. The heme synthesis and degradation pathways: role in oxidant sensitivity. Heme oxygenase has both pro- and antioxidant properties. Free Radic Biol Med. 2000;28(2):289-309.

15. Giorgi $\mathrm{C}$, et al. Mitochondrial $\mathrm{Ca}(2+)$ and apoptosis. Cell Calcium. 2012;52(1):36-43.

16. Pinton P, Rimessi A, Romagnoli A, Prandini A, Rizzuto R. Biosensors for the detection of calcium and $\mathrm{pH}$. Methods Cell Biol. 2007;80:297-325.

17. Patergnani $\mathrm{S}$, et al. Calcium signaling around Mitochondria Associated Membranes (MAMs). Cell Commun Signal. 2011;9:19.

18. Krishnamurthy P, Xie T, Schuetz JD. The role of transporters in cellular heme and porphyrin homeostasis. Pharmacol Ther. 2007;114(3):345-358.

19. Pao SS, Paulsen IT, Saier MH. Major facilitator superfamily. Microbiol Mol Biol Rev. 1998;62(1):1-34.

20. Law CJ, Maloney PC, Wang DN. Ins and outs of major facilitator superfamily antiporters. Annu Rev Microbiol. 2008;62:289-305.

21. Severance S, Hamza I. Trafficking of heme and porphyrins in metazoa. Chem Rev. 2009; 109(10):4596-4616.

22. Chen W, Dailey HA, Paw BH. Ferrochelatase forms an oligomeric complex with mitoferrin-1 and Abcb10 for erythroid heme biosynthesis. Blood. 2010; 116(4):628-630.

23. Chen W, et al. Abcb10 physically interacts with mitoferrin-1 (Slc25a37) to enhance its stability and function in the erythroid mitochondria. Proc Natl Acad Sci U S A. 2009;106(38):16263-16268.

24. Ishiura $H$, et al. Posterior column ataxia with retinitis pigmentosa in a Japanese family with a novel mutation in FLVCR1. Neurogenetics. 2011; 12(2):117-121.

25. Rajadhyaksha AM, et al. Mutations in FLVCR1 cause posterior column ataxia and retinitis pigmentosa. Am J Hum Genet. 2011;87(5):643-654.

26. Rey MA, Prasad R, Tailor CS. The C domain in the surface envelope glycoprotein of subgroup $C$ feline leukemia virus is a second receptor-binding domain. Virology. 2008;370(2):273-284.

27. Brown JK, Fung C, Tailor CS. Comprehensive mapping of receptor-functioning domains in feline leukemia virus subgroup C receptor FLVCR1.J Virol. 2006;80(4):1742-1751.

28. Shen TL, et al. Conditional knockout of focal adhesion kinase in endothelial cells reveals its role in angiogenesis and vascular development in late embryogenesis. J Cell Biol. 2005;169(6):941-952

29. Wei G, et al. Ets 1 and Ets 2 are required for endothelial cell survival during embryonic angiogenesis. Blood. 2009;114(5):1123-1130.

30. Webster WS, Abela D. The effect of hypoxia in development. Birth Defects Res C Embryo Today. 2007; 81(3):215-228.

31. Borsook H, Ratner K, Tattrie B. Studies on erythropoiesis. II. A method of segregating immature from mature adult rabbit erythroblasts. Blood. 1969; 34(1):32-41.

32. Di Stefano P, et al. P130Cas-associated protein (p140Cap) as a new tyrosine-phosphorylated protein involved in cell spreading. Mol Biol Cell. 2004; 15(2):787-800.

33. Chiabrando D, Fiorito V, Marro S, Silengo L, Altruda F, Tolosano E. Cell-specific regulation of Ferroportin transcription following experimentally-induced acute anemia in mice [published online ahead of print August 23, 2012]. Blood Cells Mol Dis. doi:10.1016/j.bbr.2011.03.031.

34. Sinclair PR, Gorman N, Jacobs JM. Measurement of heme concentration. Curr Protoc Toxicol Chapter. 2001;8:Unit 83

35. Chae SS, Paik JH, Allende ML, Proia RL, Hla T. Regulation of limb development by the sphingosine 1-phosphate receptor S1p1/EDG-1 occurs via the hypoxia/VEGF axis. Dev Biol. 2004;268(2):441-447.

36. Socolovsky M, Nam H, Fleming MD, Haase VH, Brugnara C, Lodish HF. Ineffective erythropoiesis in Stat5a(-/-) $5 \mathrm{~b}{\left(\left(^{-/}\right)\right.}$mice due to decreased survival of early erythroblasts. Blood. 2001; 98(12):3261-3273.

37. Vargas PD, Furuyama K, Sassa S, Shibahara S. Hypoxia decreases the expression of the two enzymes responsible for producing linear and cyclic tetrapyrroles in the heme biosynthetic pathway. FEBS J. 2008;275(23):5947-5959.

38. Amendola M, Venneri MA, Biffi A, Vigna E, Naldini L. Coordinate dual-gene transgenesis by lentiviral vectors carrying synthetic bidirectional promoters. Nat Biotechnol. 2005;23(1):108-116.

39. Vigna E, et al. Robust and efficient regulation of transgene expression in vivo by improved tetracycline-dependent lentiviral vectors. Mol Ther. 2002; 5(3):252-261. 